TRANSACTIONS OF THE

AMERICAN MATHEMATICAL SOCIETY

Volume 359, Number 2, February 2007, Pages 591-603

S 0002-9947(06)03888-8

Article electronically published on July 21, 2006

\title{
A LOCAL CONJECTURE ON BRAUER CHARACTER DEGREES OF FINITE GROUPS
}

\author{
THORSTEN HOLM AND WOLFGANG WILLEMS
}

\begin{abstract}
Recently, a new conjecture on the degrees of the irreducible Brauer characters of a finite group was presented by W. Willems. In this paper we propose a 'local' version of this conjecture for blocks $B$ of finite groups, giving a lower bound for $\sum \varphi(1)^{2}$ where the sum runs through the set of irreducible Brauer characters of $B$ in terms of invariants of $B$. A slight reformulation leads to interesting open questions about traces of Cartan matrices of blocks.

We show that the local conjecture is true for blocks with one simple module, blocks of $p$-solvable groups and blocks with cyclic defect groups. It also holds for many further examples of blocks of sporadic groups, symmetric groups or groups of Lie type. Finally we prove that the conjecture is true for blocks of tame representation type.
\end{abstract}

\section{INTRODUCTION}

Representation theory of finite groups is an area which is characterized by an enormous number of important long-standing open problems. Many of them deal with ordinary or modular characters, so character theory still is one of the most important and fundamental parts of representation theory. The key aim in this area is to find intimate relations between the numerical invariants given by characters and the structure of the group. Actually, R. Brauer's main motivation for introducing modular characters was to study the structure of finite simple groups.

In this paper we are going to study some of the most important numerical invariants in modular representation theory, namely degrees of irreducible Brauer characters. The main aim is to propose a conjecture relating the degrees of the irreducible characters belonging to a block to some well-known invariants of the block. This can be considered as a block version of a conjecture recently presented in 17 for finite groups. In order to state the conjecture we need to introduce some notation.

Let $G$ be a finite group and let $p$ be a prime dividing the order of $G$. Moreover, let $B$ be a $p$-block of $G$ with defect group $D$ where the underlying field of characteristic $p$ is assumed to be algebraically closed. Furthermore let $\operatorname{Irr}_{p}(B)=\left\{\varphi_{1}, \ldots, \varphi_{l(B)}\right\}$ denote the set of irreducible Brauer characters belonging to $B$, and $\operatorname{Irr}_{p}(G)$ the set of all irreducible Brauer characters of $G$.

Received by the editors April 25, 2004 and, in revised form, October 28, 2004.

2000 Mathematics Subject Classification. Primary 20C20; Secondary 15A18, 15A36, 16G60, $20 \mathrm{C} 05$.

Key words and phrases. Brauer character, block of finite group, Cartan matrix, PerronFrobenius eigenvalue.

(C)2006 American Mathematical Society Reverts to public domain 28 years from publication 
In [17 the second author proposed the following conjecture relating the degrees of the irreducible Brauer characters of a finite group $G$ to the $p^{\prime}$-part of the order of $G$ (i.e. the largest divisor of the order of $G$ not divisible by $p$ ).

Global Conjecture ([17]). For any finite group $G$ we have

$$
|G|_{p^{\prime}} \leq \sum_{\varphi \in \operatorname{Irr}_{p}(G)} \varphi(1)^{2}
$$

with equality if and only if the Sylow p-subgroup of $G$ is normal.

This conjecture is known to be true for $p$-solvable groups, groups with a cyclic Sylow $p$-subgroup, groups of Lie type in the defining characteristic, and asymptotically in many cases in non-defining characteristic, and many more examples (see [17. for details).

The main aim of this paper is to develop a 'local' version of this conjecture, i.e. a version of this conjecture for blocks of finite groups. The crucial aspect is to find a good replacement for the left-hand side, which only depends on the block and its invariants, but not on the group to which the block belongs.

We propose the following local version, relating the degrees of the irreducible Brauer characters to well-known invariants of the block. By $\operatorname{dim} B$ we denote the dimension of a block $B$ as vector space over the ground field.

Local Conjecture. Let $B$ be a p-block of a finite group, and let $D$ be its defect group. Then we have

$$
\frac{\operatorname{dim} B}{l(B)|D|} \leq \sum_{\varphi \in \operatorname{Irr}_{p}(B)} \varphi(1)^{2}
$$

with equality if and only if $l(B)=1$.

When experimentally computing examples one observes that in most cases the right-hand side becomes far larger than the left-hand side, and at first glance one is tempted to replace the right-hand side of (2) by the stronger bound

$$
\max _{\varphi \in \operatorname{Irr}_{p}(B)} \varphi(1)^{2} .
$$

However, as Christine Bessenrodt pointed out to us, this does not hold true in general, as the next example shows.

Example. Let $G=\mathrm{S}_{10}$ be the symmetric group on 10 letters and let $p=2$. By 9, the group $G$ has a 2-block of defect 3 with two irreducible Brauer characters of degrees 128 and 160, respectively. The degrees of the irreducible classical characters are $160,160,448,288$ and 288 . Thus

$$
\frac{\operatorname{dim} B}{l(B)|D|}=26112
$$

but

$$
\max _{\varphi \in \operatorname{Irr}_{p}(B)} \varphi(1)^{2}=25600 .
$$

We would like to mention that there are other examples in characteristic 2 , but we are not aware of any such example in odd characteristic.

Denote by $l(G)$ the number of $p^{\prime}$-conjugacy classes of $G$ (i.e. conjugacy classes of $G$ of elements whose order is not divisible by $p$ ). By a classical result of Brauer, 
$l(G)$ equals the number of irreducible Brauer characters of $G$. With this notation the following is obviously a weak version of the Global Conjecture.

Weak Global Conjecture. For any finite group $G$ we have

$$
\frac{|G|_{p^{\prime}}}{l(G)} \leq \max _{\varphi \in \operatorname{Irr}_{p}(G)} \varphi(1)^{2}
$$

with equality if and only if $G=O_{p}(G) \times O_{p^{\prime}}(G)$, where $O_{p^{\prime}}(G)$ is abelian.

At this moment, in either form the conjectures seem to be difficult to prove in general. Still, in this paper we are going to show that the Local Conjecture is true for various important classes of blocks of finite groups. The results can be summarized as follows.

Theorem 1. Let $B$ be a p-block of a finite group $G$ with defect group D, and let $l(B)$ be the number of irreducible Brauer characters. Then we have

$$
\frac{\operatorname{dim} B}{l(B)|D|} \leq \max _{\varphi \in \operatorname{Irr}_{p}(B)}\left\{\varphi(1)^{2}\right\}
$$

if one of the following holds:

(a) $l(B)=1$, with equality.

(b) $G$ is p-solvable.

Equality holds in (3) if and only if the following holds:

(i) All irreducible Brauer characters of $B$ have the same degree.

(ii) $\frac{|G|_{p}}{|D|}=\varphi(1)_{p}$ for all $\varphi \in \operatorname{Irr}_{p}(B)$.

(c) $D$ is cyclic.

Equality holds in (3) if the corresponding Brauer tree is a star with exceptional vertex in the center.

In particular, in all these cases the Local Conjecture holds true.

Moreover, in addition to these classes of blocks we checked many other examples for which the Local Conjecture is true, giving further evidence for its validity. Among these examples are many blocks of sporadic simple groups, blocks of (small) symmetric or alternating groups, and certain blocks of groups of Lie type.

For proving the Local Conjecture we have the following interesting approach via linear algebra. Namely, the following close relation to Cartan matrices will be proved in Section 2

Proposition 2. Let $B$ be a p-block of a finite group, and let $C$ be the Cartan matrix of $B$ with trace $\operatorname{tr} C$. Then the following holds.

(a) We have

$$
\frac{\operatorname{dim} B}{\operatorname{tr} C} \leq \sum_{\varphi \in \operatorname{Irr}_{p}(B)} \varphi(1)^{2}
$$

and equality holds if and only if $l(B)=1$.

(b) If $\operatorname{tr} C \leq l(B)|D|$, then the Local Conjecture holds for $B$.

This linear algebra approach seems to be helpful where the Cartan invariants are known but not the degrees of the irreducible Brauer characters. In this way we can prove the following result for blocks of tame representation type based on Erdmann's classification of their Cartan matrices. 
Theorem 3. The Local Conjecture holds for all blocks of tame representation type (i.e. for all 2-blocks with defect group dihedral, semidihedral or generalized quaternion).

We are not aware of any example of a block such that $\operatorname{tr} C \leq l(B)|D|$ does not hold. Actually, we strongly believe that this inequality will be true in general. Note that the inequality is not at all obvious since there are examples of blocks where the diagonal entries in the Cartan matrix are larger than $|D|$. The first example was given by Landrock [11, disproving Problem 22 of Brauer's famous list 11. Even more striking examples were found by Chastkofsky and Feit [6] they showed that for the principal 2-blocks of the Suzuki groups $\operatorname{Suz}\left(2^{m}\right)$ the first diagonal Cartan entry is asymptotically $|D|^{3 / 2}$.

Further evidence for the validity of our global conjectures is provided by analogies between degrees of irreducible characters and lengths of conjugacy classes. Such analogies have been observed by several authors (see for instance [8]). The statements about conjugacy class lengths which correspond to our Global Conjecture and Weak Global Conjecture on character degrees can actually be proved. We plan to address this issue in a separate note.

\section{Discussions around the COnjectures}

In this section we collect some of the evidence we have supporting the Local Conjecture, and discuss various aspects around it.

1.1. Blocks with one simple module. Let $B$ be a block with one simple module, i.e. $l(B)=1$. The validity of the Local Conjecture in this case is a classical result of R. Brauer, stating that in the case $l(B)=1$ equality holds in (2) (see 2, Theorems 2 and 4$)$. This proves Theorem 1(a).

For an arbitrary block $B$, Brauer showed in 2 that the $p$-part of $\frac{\operatorname{dim} B}{|D|}$ divides the $p$-part of $\sum_{\varphi \in \operatorname{Irr}_{p}(B)} \varphi(1)^{2}$. Thus equality in (2) forces $p \nmid l(B)$. But the 'only if' part of the characterization of equality remains open.

1.2. It might be tempting to try to improve the left-hand side of the inequality in the Local Conjecture. We shall see below that for some interesting classes of blocks one can actually show that

$$
\frac{\operatorname{dim} B}{|D|} \leq \sum_{\varphi \in \operatorname{Irr}_{p}(B)} \varphi(1)^{2},
$$

which obviously is stronger than the statement in the Local Conjecture for $B$.

But the following examples show that the factor $l(B)$ in the Local Conjecture can in general not be dropped and not be replaced by any constant.

Let $G=\mathrm{A}_{5}$ denote the alternating group on 5 letters and let $B$ be the principal 2-block of $G$. Then $B$ contains three irreducible Brauer characters of degrees 1,2 and 2 , respectively. The corresponding projective characters are of degree $12,8,8$. Thus we obtain

$$
\frac{\operatorname{dim} B}{|D|}=\frac{44}{4}=11>9=1+4+4=\sum_{\varphi \in \operatorname{Irr}_{2}(B)} \varphi(1)^{2} .
$$

Now we take $n$ copies of $G=\mathrm{A}_{5}$ and consider the principal 2-block $B^{(n)}$ of the direct product $G^{(n)}=G \times \ldots \times G$. Clearly $B^{(n)}$ has defect group $D^{(n)}=D \times \ldots \times D$. 
It is not hard to compute that

$$
\frac{\operatorname{dim} B^{(n)}}{\left|D^{(n)}\right|}=11^{n}>9^{n}=\sum_{\varphi \in \operatorname{Irr}_{2}\left(B^{(n)}\right)} \varphi(1)^{2} .
$$

Thus the factor $l(B)$ in the Local Conjecture cannot be replaced by a constant.

Similar examples are provided by the maximal non-principal 3-block of $6 \mathrm{~A}_{7}$, and by the principal 2-block of $\mathrm{SL}(2,5)$, showing that the above phenomenon depends on neither the prime 2 nor on $D$ being abelian.

1.3. We would like to mention that equality in (4) does not imply $l(B)=1$, as the following example shows.

Let $G$ be a finite group with a normal Sylow $p$-subgroup $P$. Furthermore let $C_{G}(P) \leq P$. Thus $G$ is a $p$-solvable group with exactly one $p$-block (see [5], Chap. $\mathrm{V}, 3.11$ ). Since $P$ is normal in $G$ it is contained in the kernel of any irreducible representation in characteristic $p$. Thus we have $p \nmid \varphi(1)$ for all $\varphi \in \operatorname{Irr}_{p}(G)$. A result of Fong (see [5], Chap. X, 3.2) yields $\Phi(1)=|P| \varphi(1)$, where $\Phi$ denotes the projective character corresponding to $\varphi$. Thus equality holds in (4), but $l(B)>1$ if $P \neq G$.

1.4. Blocks of $p$-solvable groups. Let $B$ be a block of a $p$-solvable group $G$. We are going to show that the inequality (4) holds for $B$, which implies the Local Conjecture for blocks of $p$-solvable groups.

Let $\Phi_{\varphi}$ denote the projective character corresponding to the irreducible Brauer character $\varphi$. Again by Fong's result we have $\Phi_{\varphi}(1)=|G|_{p} \varphi(1)_{p^{\prime}}$ (see [5], Chap. X, 3.2 ) and, by a result of Brauer, we have $\frac{|G|_{p}}{|D|} \mid \varphi(1)$ (see [5], Chap. IV, 4.5). Thus we get

$$
\frac{\operatorname{dim} B}{|D|}=\frac{1}{|D|} \sum_{\varphi \in \operatorname{Irr}_{p}(B)} \varphi(1) \Phi_{\varphi}(1)=\frac{|G|_{p}}{|D|} \sum_{\varphi \in \operatorname{Irr}_{p}(B)} \varphi(1) \varphi(1)_{p^{\prime}} \leq \sum_{\varphi \in \operatorname{Irr}_{p}(B)} \varphi(1)^{2} .
$$

For proving Theorem 1(b) it remains to characterize equality in (3). From the discussions above we have

$$
\frac{\operatorname{dim} B}{|D|} \leq \sum_{\varphi \in \operatorname{Irr}_{p}(B)} \varphi(1)^{2} \leq l(B) \max _{\varphi \in \operatorname{Irr}_{p}(B)}\left\{\varphi(1)^{2}\right\}
$$

where the first inequality is an equality if and only if $\frac{|G|_{p}}{|D|}=\varphi(1)_{p}$ for all $\varphi \in$ $\operatorname{Irr}_{p}(B)$. Clearly, the second inequality becomes an equality if and only if all irreducible Brauer characters have the same degree. This proves Theorem 1(b).

1.5. Connections to classical open problems. Let $k(B)$ denote the number of ordinary irreducible characters in an arbitrary block $B$, with defect group $D$. Clearly $l(B) \leq k(B)$. Problem 20 of Brauer's famous list [1 asks whether always $k(B) \leq|D|$. If we assume that this has an affirmative answer we get $l(B)|D| \leq|D|^{2}$, and our Local Conjecture leads to

$$
\frac{\operatorname{dim} B}{|D|^{2}} \leq \sum_{\varphi \in \operatorname{Irr}_{p}(B)} \varphi(1)^{2} .
$$

This would obviously hold true if

$$
\Phi_{\varphi}(1) \leq|D|^{2} \varphi(1) \text { for all } \varphi \in \operatorname{Irr}_{p}(B),
$$


where $\Phi_{\varphi}$ is the projective character corresponding to $\varphi$. Unfortunately, there are examples for which (6) is wrong. For instance, in characteristic 3 we have $\Phi_{\varphi}(1)=$ 99 for the trivial character $\varphi$ of the alternating group $\mathrm{A}_{7}$ and $|D|^{2} \varphi(1)=81$.

1.6. What are the connections between the Global Conjecture from 17 and the Local Conjecture? An affirmative answer to the Local Conjecture would also prove a weaker version of the Global Conjecture. In fact, if $l(G)$ denotes the number of $p^{\prime}$-conjugacy classes of $G$, then by summing up (2) over all blocks $B$ of $G$ we would get

$$
\frac{|G|_{p^{\prime}}}{l(G)} \leq \sum_{\varphi \in \operatorname{Irr}_{p}(G)} \varphi(1)^{2}
$$

with equality if and only if $G$ is a $p$-group. The latter fact can be seen as follows. The Local Conjecture implies

$$
|G|=\sum_{B} \operatorname{dim} B \leq \sum_{B} l(B)|D| \sum_{\varphi \in \operatorname{Irr}_{p}(B)} \varphi(1)^{2} \leq l(G)|G|_{p} \sum_{\varphi \in \operatorname{Irr}_{p}(G)} \varphi(1)^{2} .
$$

Thus equality in (7) forces equality in (2) for each block $B$, hence $l(B)=1$, and $l(B)=l(G)$. Thus the trivial character is the only irreducible Brauer character and therefore $G$ has only one conjugacy class of $p^{\prime}$-elements. This shows that $G$ is a $p$-group. On the other hand, if $G$ is a $p$-group, then obviously equality holds in (17).

(Note that in general $\Phi_{\varphi}(1) \not \leq|P| l(G) \varphi(1)$, where $P$ is a Sylow $p$-subgroup of $G$. Take the projective character corresponding to the trivial character for the smallest Suzuki group in characteristic 2.)

1.7. As mentioned in the Introduction we do not know any example in odd characteristic of a block $B$ for which

$$
\frac{\operatorname{dim} B}{l(B)|D|} \leq \max _{\varphi \in \operatorname{Irr}_{p}(B)} \varphi(1)^{2}
$$

does not hold. So let us assume that $p \neq 2$ and that (8) is true for all blocks. If we now sum up (8) over all blocks we get the Weak Global Conjecture

$$
\frac{|G|_{p^{\prime}}}{l(G)} \leq \max _{\varphi \in \operatorname{Irr}_{p}(B)} \varphi(1)^{2} .
$$

Moreover, and here $p \neq 2$ is crucial for what follows, equality holds if and only if $G=O_{p}(G) \times O_{p^{\prime}}(G)$, where $O_{p^{\prime}}(G)$ is abelian. Thus the assumption (8) for all blocks $B$ leads to a complete proof of the Weak Global Conjecture in the case of odd characteristic.

Indeed if $G=O_{p}(G) \times O_{p^{\prime}}(G)$ where $O_{p^{\prime}}(G)$ is abelian, then equality holds in (9). In order to prove the converse suppose that equality holds in (9). First we prove by induction that $G$ is $p$-solvable. We put $H=O_{p^{\prime}}(G)$ and $\bar{G}=G / H$. Since the group algebra of $\bar{G}$ is a direct summand of the group algebra of $G$ over the underlying field, one can show that

$$
\frac{|\bar{G}|_{p^{\prime}}}{l(\bar{G})}=\max _{\varphi \in \operatorname{Irr}_{p}(\bar{G})}\left\{\varphi(1)^{2}\right\} .
$$


If $H \neq\langle 1\rangle$, then by induction $\bar{G}$ is $p$-solvable. Hence, $G$ is $p$-solvable. Next we assume that $O_{p}(G) \neq\langle 1\rangle$. If $\hat{G}=G / O_{p}(G)$, then equality in (9) implies that

$$
\frac{|\hat{G}|_{p^{\prime}}}{l(\hat{G})}=\max _{\varphi \in \operatorname{Irr}_{p}(\hat{G})}\left\{\varphi(1)^{2}\right\}
$$

and the induction argument yields again that $\hat{G}$ is $p$-solvable. Therefore, $G$ is also $p$-solvable. Finally, we may assume that $G$ has a minimal normal subgroup, say $N$, which is a direct product of isomorphic non-abelian simple groups whose orders are divisible by $p$. The equality in (9) implies that all blocks of $G$ are of maximal defect. Thus, by Knörr's theorem (see [13, 9.26), all blocks of $N$ are of maximal defect. By a result of Michler (see [12, Theorem 5.3), a finite non-abelian simple group has blocks of different defects for $p \neq 2$. Thus we have a contradiction in this case, and the claim that $G$ is $p$-solvable has been proved.

Next we show that $G$ is an abelian $p^{\prime}$-group. Since

$$
|G|_{p^{\prime}}=\sum_{\varphi \in \operatorname{Irr}_{p}(G)} \varphi(1)_{p^{\prime}} \varphi(1) \leq l(G) \max _{\varphi \in \operatorname{Irr}_{p}(G)}\left\{\varphi(1)^{2}\right\}=|G|_{p^{\prime}},
$$

all $\varphi$ are of degree 1. In particular

$$
G^{\prime} \leq \cap_{\varphi \in \operatorname{Irr}_{p}(G)} \operatorname{Ker} \varphi=O_{p}(G),
$$

where $G^{\prime}$ denotes the commutator subgroup of $G$. Thus $G$ has a normal Sylow $p$-subgroup with abelian factor group. The equation

$$
\frac{|G|_{p^{\prime}}}{l(G)}=\max _{\varphi \in \operatorname{Irr}_{p}(G)}\left\{\varphi(1)^{2}\right\}=1
$$

shows that all $p^{\prime}$-conjugacy classes have length 1 . Thus a Hall $p^{\prime}$-subgroup of $G$ is central in $G$, which proves the assertion.

\section{Eigenvalues and Brauer characters}

Associated to any $p$-block $B$ is the Cartan matrix $C=C_{B}$. If $\langle\cdot, \cdot\rangle$ denotes the Euclidean inner product on $\mathbb{R}^{l}$ and $\bar{\varphi}=\left(\varphi_{1}(1), \ldots, \varphi_{l}(1)\right)$ the dimension vector, then the dimension of the block can be expressed as $\operatorname{dim} B=\langle C \bar{\varphi}, \bar{\varphi}\rangle$. If $\rho(C)$ denotes the Perron-Frobenius eigenvalue of $C$, i.e.

$$
\rho(C)=\max \{|\lambda| \mid \lambda \text { is an eigenvalue of } C\},
$$

then, since $C$ is a Hermitian matrix,

$$
\frac{\langle C \bar{\varphi}, \bar{\varphi}\rangle}{\langle\bar{\varphi}, \bar{\varphi}\rangle} \leq \rho(C) .
$$

In particular we have the following result.

Proposition 2.1 (Kiyota-Wada [10]).

$$
\frac{\operatorname{dim} B}{\rho(C)}=\frac{\langle C \bar{\varphi}, \bar{\varphi}\rangle}{\rho(C)} \leq\langle\bar{\varphi}, \bar{\varphi}\rangle=\sum_{\varphi \in \operatorname{Irr}_{p}(B)} \varphi(1)^{2} .
$$

In order to prove our Local Conjecture it therefore suffices to show that $\rho(C) \leq$ $l(B)|D|$ holds for any block. Usually, $\rho(C)$ is hard to determine, and there is no general upper bound known which only depends on invariants of $B$, and not on the group. Moreover, equality in (10) does not seem to be easy to characterize. In fact, suppose equality holds in (10). Since $C$ is Hermitian, this implies $C \bar{\varphi}=\rho(C) \bar{\varphi}$. 
Thus if $\Phi_{\varphi}$ denotes the projective character corresponding to $\varphi$, then we have $\Phi_{\varphi}(1)=\rho(C) \varphi(1)$. By Theorem 3 of [2] we have $\operatorname{gcd}\left(\Phi_{\varphi}(1) \mid \varphi \in \operatorname{Irr}_{p}(B)\right)=p^{a} u_{B}$, where $|G|_{p}=p^{a}$ and $u_{B}$ is an integer not divisible by $p$. Moreover, by Theorem 2 of the same paper we have $\operatorname{gcd}\left(\varphi(1) \mid \varphi \in \operatorname{Irr}_{p}(B)\right)=p^{a-d} u_{B}$, where $|D|=p^{d}$. Since $\Phi_{\varphi}(1)=\rho(C) \varphi(1)$ we conclude that $\rho(C)=|D|$. On the other hand if $\Phi_{\varphi}(1)=|D| \varphi(1)$ for all $\varphi \in \operatorname{Irr}_{p}(B)$, then equality holds in (10).

Such situations occur for all blocks if $G$ is $p$-solvable of $p$-length 1 . In particular, equality in (10) does not imply $l(B)=1$ in general.

Despite the difficulties in general, for blocks of $p$-solvable groups we have the following characterization of equality in (10).

Proposition 2.2. Let $G$ be a p-solvable group. For the principal p-block $B$ the following statements are equivalent:

(a) $\frac{\operatorname{dim} B}{\rho(C)}=\sum_{\varphi \in \operatorname{Irr}_{p}(B)} \varphi(1)^{2}$, i.e. equality holds in (10).

(b) $G$ is of p-length 1 .

Proof. As mentioned above, (b) implies (a) (see [14, Satz 4.2). To prove the other direction let $P$ denote a Sylow $p$-subgroup of $G$. By the remarks preceding the proposition we have $\Phi_{\varphi}(1)=|P| \varphi(1)$ for all $\varphi \in \operatorname{Irr}_{p}(B)$. This implies $\Phi_{\varphi}=\varphi \Phi_{1}$, where $\Phi_{1}$ corresponds to the trivial character. If $p \mid \varphi(1)$, then the multiplicity of the trivial character in $\varphi \bar{\varphi}$ is at least 2 (see [7], Chap. VII, $8.5 \mathrm{~d}$ )). Thus

$$
2 \leq\left(\Phi_{1}, \varphi \bar{\varphi}\right)=\left(\Phi_{1} \varphi, \varphi\right)=\left(\Phi_{\varphi}, \varphi\right)=1,
$$

a contradiction. So all irreducible Brauer characters in $B$ are of $p^{\prime}$-degree. Since $G$ is assumed to be $p$-solvable and $B$ is the principal block, we conclude that $B$ is isomorphic to the group algebra of $G / O_{p^{\prime}}(G)$. The condition $p \nmid \varphi(1)$ for all $\varphi$ in $B$ implies that $G / O_{p^{\prime}}(G)$ has a normal Sylow $p$-subgroup (a well-known result of Wallace, see [16 or [3]). Thus $G$ is of $p$-length 1 .

Our next aim is to establish a linear algebra criterion for proving the Local Conjecture, namely Proposition 2 as stated in the Introduction. We begin by recalling some useful fundamental facts about Cartan matrices of blocks.

Lemma 2.3. The Cartan matrix of any p-block $B$ is positive definite.

Proof. If $D$ denotes the decomposition matrix of $B$, then $C=D^{T} D$. Then we have $(C x, x)=\left(D^{T} D x, x\right)=(D x, D x)$ for all $x \in \mathbb{R}^{l}$, where $l=l(B)$. If $(D x, D x)=0$, then $D x=0$ and therefore $C x=0$. But $C$ is regular, hence $x=0$. This proves that $(C x, x)>0$ for all $0 \neq x \in \mathbb{R}^{l}$.

Corollary 2.4. If $C$ is the Cartan matrix of a p-block, then

$$
\rho(C) \leq \operatorname{tr} C .
$$

Proof. Since $C$ is positive definite by Lemma 2.3, all eigenvalues of $C$ are positive, and they sum up to the trace of $C$. In particular $\rho(C) \leq \operatorname{tr} C$.

Proposition 2.5. Let $B$ be a p-block of a finite group, with Cartan matrix $C$. Then

$$
\frac{\operatorname{dim} B}{\operatorname{tr} C} \leq \sum_{\varphi \in \operatorname{Irr}_{p}(B)} \varphi(1)^{2}
$$

and equality holds if and only if $l(B)=1$. 
Proof. By Proposition 2.1 and Corollary 2.4 we have

$$
\frac{\operatorname{dim} B}{\operatorname{tr} C} \leq \frac{\operatorname{dim} B}{\rho(C)} \leq \sum_{\varphi \in \operatorname{Irr}_{p}(B)} \varphi(1)^{2} .
$$

Suppose equality holds, i.e. $\operatorname{tr} C=\rho(C)$. Since all eigenvalues are positive, and their sum is $\operatorname{tr} C$, we deduce that $l(B)=1$.

The above proposition gives a possible linear algebra approach to proving the Local Conjecture, provided the following question has an affirmative answer.

Question 2.6. Let $B$ be a $p$-block of a finite group, with defect group $D$ and Cartan matrix $C$. Is it true that

$$
\operatorname{tr} C \leq l(B)|D| ?
$$

Since $C$ is positive definite we have

$$
\operatorname{det} C \leq \prod_{\varphi \in \operatorname{Irr}_{p}(B)} c_{\varphi \varphi}
$$

hence

$$
l \sqrt[l]{\operatorname{det} C} \leq l \sqrt[l]{\prod_{\varphi \in \operatorname{Irr}_{p}(B)} c_{\varphi \varphi}} \leq \sum_{\varphi \in \operatorname{Irr}_{p}(B)} c_{\varphi \varphi}=\operatorname{tr} C .
$$

Thus the truth of (11) would imply $\sqrt[l]{\operatorname{det} C} \leq|D|$. This is indeed the case, as one can see using well-known properties of elementary divisors of $C$.

\section{BLOCKS WITH CYCLIC DEFECT GROUPS}

This section deals with blocks with cyclic defect group and contains a proof of Theorem 1(c). The proof is very much based on the well-known structure theory of these blocks, and it also follows from work of Kiyota and Wada 10. For the sake of completeness and for the convenience of the reader we include a direct proof here. We actually show the following stronger statement.

Proposition 3.1. For a p-block $B$ with cyclic defect group $D$ we have

$$
\frac{\operatorname{dim} B}{|D|} \leq \sum_{\varphi \in \operatorname{Irr}_{p}(B)} \varphi(1)^{2}
$$

with equality if and only if the corresponding Brauer tree of $B$ is a star with exceptional vertex, if there is one, in the center.

Proof. Let $e$ denote the inertial index of $B$. In other words $e$ is the number $l(B)$ of irreducible Brauer characters in $B$. If we put $m=\frac{|D|-1}{e}$, then the shape of the Brauer tree yields

$$
0 \leq C=\left(c_{i, j}\right) \leq\left(\begin{array}{ccc}
m+1 & & \\
& \ddots & m \\
m & & m+1
\end{array}\right)
$$

for the Cartan matrix $C$ of $B$ where $\leq$ for matrices means $\leq$ in each position. If we put $\bar{\varphi}=\left(\varphi_{1}(1), \ldots, \varphi_{e}(1)\right)$ and $\overline{1}=(1, \ldots, 1)$, then by the Cauchy-Schwarz inequality

$$
|\langle\bar{\varphi}, \overline{1}\rangle|^{2} \leq\langle\bar{\varphi}, \bar{\varphi}\rangle\langle\overline{1}, \overline{1}\rangle,
$$


hence

$$
\left(\sum_{i=1}^{e} \varphi_{i}(1)\right)^{2} \leq e \sum_{i=1}^{e} \varphi(1)^{2} .
$$

Thus we get

$$
\begin{array}{rlr}
\operatorname{dim} B & =\sum_{i=1}^{e} \varphi_{i}(1) \Phi_{i}(1) & \\
& =\sum_{i, j=1}^{e} \varphi_{i}(1) c_{i, j} \varphi_{j}(1) & \\
& \leq m \sum_{i, j=1}^{e} \varphi_{i}(1) \varphi_{j}(1)+\sum_{i=1}^{e} \varphi_{i}(1)^{2} & \\
& =m \sum_{i=1}^{e} \varphi_{i}(1) \sum_{j=1}^{e} \varphi_{j}(1)+\sum_{i=1}^{e} \varphi_{i}(1)^{2} & \\
& \leq m e \sum_{i=1}^{e} \varphi_{i}(1)^{2}+\sum_{i=1}^{e} \varphi_{i}(1)^{2} & \text { by }(*) \\
& =(m e+1) \sum_{i=1}^{e} \varphi_{i}(1)^{2} & \\
& =|D| \sum_{i=1}^{e} \varphi_{i}(1)^{2} . &
\end{array}
$$

Suppose equality holds in (12). It follows that

$$
C=\left(c_{i, j}\right)=\left(\begin{array}{ccc}
m+1 & & \\
m & \ddots & m \\
& & m+1
\end{array}\right) \text {. }
$$

This means that the Brauer tree is a star with the exceptional vertex (if there is one) in the center.

\section{Cartan matrices of tame Blocks}

In this section we give a proof of our Local Conjecture for blocks of tame representation type, i.e. for all 2-blocks of finite groups having a dihedral, semidihedral or generalized quaternion defect group. For the proof we use the linear algebra approach outlined in Section 2, namely we are going to show that the trace of the Cartan matrix can be bounded above in terms of the order of the defect group. The Cartan matrices of tame blocks are known due to the extraordinary classification of K. Erdmann of tame blocks up to Morita equivalence 44.

Blocks of tame representation type have at most three simple modules. Since our conjectures are true for blocks with one simple module, we can restrict our attention to the blocks with two or three simple modules. We refer to [4 for background on tame blocks, and also for details and notations.

Proposition 4.1. Let $B$ be a block of a finite group of tame representation type, with defect group D. Then for the trace of the Cartan matrix $C$ of $B$ the following holds:

(a) If $B$ has two simple modules, then we have $\operatorname{tr} C \leq \frac{5}{4}|D|+2$.

(b) If $B$ has three simple modules, then we have $\operatorname{tr} C \leq \frac{3}{2}|D|+4$.

Proof. (a) From Erdmann's classification we see that such a block $B$ has a Cartan matrix of the form

$$
\left(\begin{array}{cc}
4 k & 2 k \\
2 k & k+r
\end{array}\right)
$$


with natural numbers $k$ and $r$, where $\{k, r\}=\left\{1, \frac{|D|}{4}\right\}$ or $\{k, r\}=\left\{2, \frac{|D|}{4}\right\}$. For any possiblity of $k$ and $r$ we obtain that $\operatorname{tr} C \leq \frac{5}{4}|D|+2$ (note that $|D| \geq 8$, since a block with Klein four defect group cannot have two simple modules).

(b) There are various families of algebras in Erdmann's classification which (may) contain blocks of finite groups. They are denoted $D(3 \mathcal{A})_{1}, D(3 \mathcal{B})_{1}$ and $D(3 \mathcal{K})$ in the dihedral case, $S D(3 \mathcal{A})_{1}, S D(3 \mathcal{B})_{1}, S D(3 \mathcal{C})_{2}$ and $S D(3 \mathcal{D})$ in the semidihedral case, and $Q(3 \mathcal{A})_{2}, Q(3 \mathcal{B})$ and $Q(3 \mathcal{K})$ in the quaternion case.

The families $D(3 \mathcal{A})_{1}$ and $Q(3 \mathcal{A})_{2}$ have Cartan matrices of the form

$$
\left(\begin{array}{ccc}
4 k & 2 k & 2 k \\
2 k & k+a & k \\
2 k & k & k+a
\end{array}\right),
$$

where $k \in \mathbb{N}$ and $a \in\{1,2\}$. Blocks of finite groups only occur for $k=\frac{|D|}{4}$. In particular, $\operatorname{tr} C \leq \frac{3}{2}|D|+4$.

The algebras $D(3 \mathcal{K})$ and $Q(3 \mathcal{K})$ have Cartan matrices of the form

$$
\left(\begin{array}{ccc}
2 a & a & a \\
a & k+a & k \\
a & k & k+a
\end{array}\right),
$$

where $k \in \mathbb{N}$ and $a \in\{1,2\}$. For blocks of finite groups we have $k=\frac{|D|}{4}$. In particular $\operatorname{tr} C \leq \frac{|D|}{2}+8 \leq \frac{3}{2}|D|+4$ (since $|D| \geq 4$ for tame blocks).

The algebras in the families $S D(3 \mathcal{B})_{1}, S D(3 \mathcal{D})$ and $Q(3 \mathcal{B})$ all have Cartan matrices of the form

$$
\left(\begin{array}{ccc}
4 & 2 & 2 \\
2 & s+1 & 1 \\
2 & 1 & 3
\end{array}\right)
$$

for a natural number $s \geq 2$. Blocks of finite groups only occur for $s=\frac{|D|}{4} \geq 2$. In particular we get that $\operatorname{tr} C \leq \frac{|D|}{4}+8 \leq \frac{3}{2}|D|+4$.

The algebras in the family $D(3 \mathcal{B})_{1}$ have Cartan matrix of the form

$$
\left(\begin{array}{ccc}
4 & 2 & 2 \\
2 & s+1 & 1 \\
2 & 1 & 2
\end{array}\right)
$$

for some $s \geq 1$. Blocks of finite groups occur for the parameter $s=\frac{|D|}{4}$, hence $\operatorname{tr} C \leq \frac{|D|}{4}+7$ for blocks.

The algebras in the family $S D(3 \mathcal{A})_{1}$ have Cartan matrix of the form

$$
\left(\begin{array}{ccc}
4 k & 2 k & 2 k \\
2 k & k+1 & k \\
2 k & k & k+2
\end{array}\right)
$$

for some natural number $k$. Blocks of finite groups occur for parameters $k=\frac{|D|}{4}$, i.e. $\operatorname{tr} C \leq \frac{3}{2}|D|+3$ for blocks in this family.

There are two more families for which it is still an open question whether blocks of finite groups actually occur in these families. 
The Cartan matrices of algebras in the family $S D(3 \mathcal{H})$ are of the form

$$
\left(\begin{array}{ccc}
3 & 2 & 1 \\
2 & s+2 & s \\
1 & s & s+1
\end{array}\right)
$$

for some $s \geq 2$. If blocks occur, then they occur for $s=\frac{|D|}{4}$. In particular, for blocks we have $\operatorname{tr} C \leq \frac{|D|}{2}+5 \leq \frac{3}{2}|D|+4$.

The Cartan matrices for algebras in the family $S D(3 \mathcal{C})$ are of the form

$$
\left(\begin{array}{ccc}
k+s & k & k \\
k & k+1 & k-1 \\
k & k-1 & k+1
\end{array}\right) .
$$

If blocks of finite groups occur, then we have $\{k, s\}=\left\{2, \frac{|D|}{4}\right\}$. For $k=2, s=\frac{|D|}{4}$ we get $\operatorname{tr} C \leq \frac{|D|}{4}+8$, in the other case we get $\operatorname{tr} C \leq \frac{3}{4}|D|+4$.

This completes the proof of the proposition.

As an immediate consequence of the above proposition, we obtain that if $B$ is a block of a finite group with tame representation type, then $\operatorname{tr} C \leq l(B)|D|$, where $D$ is the defect group. (Note that a block with a Klein four group as a defect group has three irreducible Brauer characters.) By Proposition 2.5 we can conclude that the Weak Conjecture holds for all tame blocks, thus proving Theorem 3.

\section{REFERENCES}

[1] R. Brauer, Representations of finite groups. Lectures on Modern Mathematics, John Wiley, New York 1963. MR0178056 (31:2314)

[2] R. Brauer, Notes on representations of finite groups, I. J. London Math. Soc. (2) 13 (1976), 162-166. MR0399240 (53:3091)

[3] P. Brockhaus, On the radical of a group algebra. J. Algebra 95 (1985), 454-472. MR0801281 (87h:20021)

[4] K. Erdmann, Blocks of Tame Representation Type and Related Algebras. Lecture Notes in Mathematics 1428, Springer (1990). MR1064107 (91c:20016)

[5] W. Feit, The representation theory of finite groups. North-Holland, Amsterdam 1982. MR.0661045 (83g:20001)

[6] L. Chastkofsky, W. Feit, On the projective characters in characteristic 2 of the groups $\operatorname{Suz}\left(2^{m}\right)$ and $S p_{4}\left(2^{n}\right)$. Inst. Hautes Études Sci. Publ. Math. No. 51 (1980), 9-35. MR0573820 (83b:20014)

[7] B. Huppert, N. Blackburn, Finite groups II, III Springer-Verlag, Berlin, Heidelberg, New York, 1982.

[8] B. Huppert, Research in representation theory at Mainz (1984-1990). In: G.O. Michler, C.M. Ringel (eds.), Representation theory of finite groups and finite-dimensional algebras. Birkhäuser, Basel, 1991. MR1112156 (92c:20011)

[9] G. James, A. Kerber, The representation theory of the symmetric group. Encyclopedia of Mathematics and Its Applications 16, Addison-Wesley 1981. MR0644144 (83k:20003)

[10] M. Kiyota, T. Wada, Some remarks on eigenvalues of the Cartan matrix in finite groups. Comm. Algebra 21(11) (1993), 3839-3860. MR.1238129 (94i:20021)

[11] P. Landrock, A counterexample to a conjecture on the Cartan invariants of a group algebra. Bull. London Math. Soc. 5 (1973), 223-224. MR 0323887 (48:2240)

[12] G. O. Michler, A finite simple group of Lie type has p-blocks with different defects. J. Algebra 104 (1986), 220-230. MR0866772 (87m:20038)

[13] G. Navarro, Characters and Blocks of Finite Groups. Lecture Notes Series 250, Cambridge University Press, Cambridge 1998. MR.1632299 (2000a:20018)

[14] W.Schwarz, Die Struktur modularer Gruppenringe endlicher Gruppen der p-Länge 1. J. Algebra 60 (1979), 51-75. MR0549098 (81a:20012) 
[15] T. Wada, A lower bound of the Perron-Frobenius eigenvalue of the Cartan matrix for a finite group. Arch. Math. 73 (1999), 407-413. MR.1725175 (2000i:20016)

[16] D.A.R. Wallace, On the radical of a group algebra. Proc. Amer. Math. Soc. 12 (1961), 133137. MR0121408 (22:12146)

[17] W. Willems, On degrees of irreducible Brauer characters. Trans. Am. Math. Soc. 357 (2005), 2379-2387. MR2140443 (2006a:20019)

Department of Pure Mathematics, University of Leeds, Leeds LS2 9JT, United KingDOM

E-mail address: tholm@maths.leeds.ac.uk

Current address: Institut für Algebra und Geometrie, Otto-von-Guericke-Universität, Postfach 4120, 39016 Magdeburg, Germany

Otto-von-Guericke-Universität, Institut für Algebra und Geometrie, Postfach 4120, 39016 Magdeburg, Germany

E-mail address: wolfgang.willems@mathematik. uni-magdeburg.de 